states out of four (the detection of one of the two states fails half of the time; Fig. 1b). Bob's photon is then buffered in a delay line (a $15 \mathrm{~km}$ fibre spool) to have the time to apply the corrective unitary corresponding to the measurement result - or to throw it away in case of failure. The Calgary team chose the simpler measurement, where only the Bell state implying no correction is detected (Fig. 1c). This leads to a success probability of only $25 \%$, but reduces the correction to a trivial post selection that can even be performed a posteriori.

A quantum network operator, traditionally called Charlie, would want to keep the expensive components in the core of the network and serve its customers, traditionally called Alice and Bob, who are operating simpler - hence cheaper terminals. Owing to its complexity specifically the cryogenics needed to operate the SNSPDs - the Bell measurement apparatus is a natural candidate to be operated by Charlie.

Interestingly, the limitations of both of these first teleportation demonstrations on metropolitan fibre networks are different, and this suggests that rapid progress can be made in the near future. In the Hefei experiment (Fig. 1b), the qubits (quantum bits of information) are carried by true single photons at a telecom wavelength, and an active feed-forward scheme is used to achieve a result close to the ideal theoretical teleportation. However, this performance comes at the price of a low rate of two teleported photons per hour, which would strongly limit its practical applications if it could not be improved.

In contrast, in the Calgary experiment (Fig. 1c), Alice's state is not encoded onto a true single photon but instead on an attenuated coherent state. This simplification imposes the use of the so-called decoy-states protocol - developed for quantum cryptography - to ensure that the process observed is really a teleportation and that the use of true single photons would lead to the same result. Instead of using $1.5 \mu \mathrm{m}$ telecom wavelengths like the Chinese team, here the state is teleported onto a $795 \mathrm{~nm}$ photon, which allows the use of more efficient photodetectors (silicon avalanche photodiodes) at Bob's side. This allows a faster teleportation rate of 17 photons per minute, two orders of magnitudes faster than in Hefei. But the a posteriori nature of the experiment also limits its immediate practical applications.

Combined, these two experiments clearly show that teleportation across metropolitan distances is technologically feasible, and undoubtedly many interesting quantum information experiments in the future will be built on this work. For the longer term, the two papers demonstrate that the possibility of quantum networks that span a city are a realistic proposition, which is an exciting vision for the future.

Frédéric Grosshans is at Laboratoire Aimé Cotton, CNRS, Université Paris-Sud, ENS Cachan, Université Paris-Saclay, 91405 Orsay, France. e-mail: frederic.grosshans@u-psud.fr

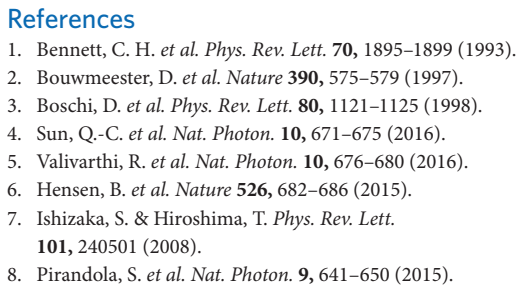

Published online: 19 September 2016

\title{
BIOPHOTONICS
}

\section{Bright sponges}

Hexactinellid sponges - also known as glass sponges because of their silica-based skeletal elements called spicules - offer an example of natural systems with notable optical properties.

Previous studies have showed that the spicules of such sponges can guide light thanks to total internal reflection. Now Hermann Ehrlich and collaborators have observed supercontinuum generation (SG) in the anchoring needle-like spicules of Sericolophus hawaiicus (pictured; left), which lives in the sea at depths of around $400 \mathrm{~m}$ in Hawaii (Adv. Opt. Mater. http:// doi.org/10.1002/adom.201600454; 2016).

First, the team investigated the structure of the spicules. Scanning electron microscopy indicated that the spicules consist of three distinct regions: a central 1- $\mu \mathrm{m}$-wide hollow channel, a surrounding solid region of highly pure silica with a thickness between 25 and $100 \mu \mathrm{m}$, and an outer region of concentric silica-chitin layers (right panels).

Second, when Ehrlich and co-workers coupled pulsed beams with optical peak intensities between 1 and $100 \mathrm{TW} \mathrm{cm}^{-2}$

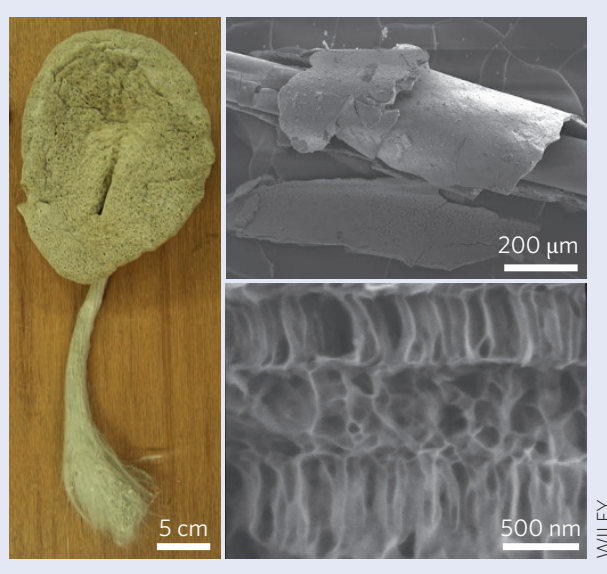

that the whole spicule served as the active medium for SG.

According to the authors, the origin of SG might be due to the composite structure of the spicules, where the high isotopic purity of the silica could favour light transmission and the chitinous matrix is thought to enhance the flexibility of the anchoring stalk, which is normally composed of hundreds of spicules. As Ehrlich told Nature Photonics, a promising feature of the spicules of S. hawaiicus is their minimal use of materials, that is, their "high strength-to-weight ratio."

Whereas the fabrication of commercial glass fibres requires very and wavelengths ranging from 650 to $900 \mathrm{~nm}$ into 30- to 50-mm-long fragments of spicules, they observed the emission of a supercontinuum with wavelengthdependent spectral width at the output. The widest spectrum was obtained for an input wavelength around $800 \mathrm{~nm}$; higher excitation intensities and longer spicules both led to increased spectral broadening, providing further evidence that white-light emission was not due to fluorescence and high temperatures, hexactinellid sponges such as S. hawaiicus produce the spicules responsible for their anchorage at temperatures close to $4^{\circ} \mathrm{C}$. The authors speculate that a better understanding of the dispersive and nonlinear properties of these natural optical fibres could facilitate the synthesis of new light-guiding materials.

GAIA DONATI 